\title{
Do tests of executive functioning predict ability to downregulate emotions spontaneously and when instructed to suppress?
}

\author{
Anett Gyurak, Madeleine S. Goodkind, and Anita Madan \\ University of California, Berkeley, California \\ Joel H. Kramer and Bruce L. Miller \\ University of California, San Francisco, California \\ AND \\ ROBERT W. LEVENSON \\ University of California, Berkeley, California
}

\begin{abstract}
Behavioral regulation is a hallmark feature of executive functioning (EF). The present study investigated whether commonly used neuropsychological test measures of EF (i.e., working memory, Stroop, trail making, and verbal fluency) were related to ability to downregulate emotion both spontaneously and when instructed to suppress emotional expressions. To ensure a wide range of EF, 24 frontotemporal lobar degeneration patients, 7 Alzheimer's patients, and 17 neurologically normal controls participated. Participants were exposed to an acoustic startle stimulus (single aversive noise burst) under three conditions: (1) unwarned, (2) warned with no instructions (to measure spontaneous emotion downregulation), and (3) warned with instructions to suppress (to measure instructed emotion downregulation). Results indicated that higher verbal fluency scores were related to greater emotion regulation (operationalized as reduction in body movement and emotional facial behavior when warned of the impending startle) in both regulation conditions. No relationships were found between emotion regulation in these conditions and the other EF measures. We conclude that, of four commonly used measures of EF, verbal fluency best indexes the complex processes of monitoring, evaluation, and control necessary for successful emotion regulation, both spontaneously and following instructions to suppress.
\end{abstract}

The ability to monitor and modify ongoing behavior is essential to many cognitive and emotional functions. A set of psychological processes collectively called executive functioning $(\mathrm{EF})$ has been associated with coordinating perceptual and motor processes in the service of behavioral goals (Lezak, Howieson, Loring, Hannay, \& Fischer, 2004; Norman \& Shallice, 1986; Smith \& Jonides, 1999). These processes allow us to do such things as participate in a conversation despite having something else on our minds, modify routine responses, learn new tasks, notice and correct inappropriate behavior, and regulate our emotional responses (Posner \& Rothbart, 2000; Stuss \& Alexander, 2000; Zelazo \& Cunningham, 2007).

It is well documented that deficits in EF can result in behaviors becoming repetitive and stimulus bound and in difficulties in successfully integrating perceptual information in cognitive tasks (Baddeley, 1986; Stuss \& Levine, 2002). However, the role of EF deficits in emotion regulation is not as well understood. Emotion regulatory processes are goal-directed behaviors, functioning to modify dynamic features of emotion such as the magnitude and duration of behavioral (i.e., expressive), experiential, and physiological responses (Gross \& Thompson, 2007). Successful emotion regulation draws heavily on EF in realms such as anticipating outcomes, planning, and executing responses (e.g., Banfield, Wyland, Macrae, Münte, \& Heatherton, 2004; Denckla, 1996). For example, in order to downregulate manifestations of fear in response to a threatening stimulus, one has to integrate perceptual cues, anticipate one's responses to these cues, devise an action plan (e.g., keep one's breathing steady and facial muscles immobile), and continuously monitor and adjust ongoing behavior.

Indeed, studies using neurologically intact populations show that EF is related to the ability to modulate emotionally laden responses, such as reducing prejudiced behaviors (von Hippel, Silver, \& Lynch, 2000), reducing biased opinions (Payne, 2005), refraining from expressing disgust in a socially unacceptable setting (von Hippel \& Gonsalkorale, 2005), and delaying gratification (Eigsti et al., 2006). Because EF is thought to be related to the integrity of the frontal lobes (e.g., Royall et al., 2002), lesion studies that report diminished emotion regulatory functioning among 
patients with circumscribed focal damage also support the idea that EF is related to emotion regulation. Specifically, researchers suggest that frontal lobe damage and resultant EF deficits compromise abilities to integrate emotional cues into decision making (e.g., Eslinger \& Damasio, 1985 ) and to evaluate the relevance of emotional cues to the task at hand (Rule, Shimamura, \& Knight, 2002).

Recent fMRI research also implicates frontal brain structures, linked to EF, in the conscious control and cognitive reappraisal of emotional stimuli (e.g., Beauregard, Lévesque, \& Bourgouin, 2001; Ochsner, Bunge, Gross, \& Gabrieli, 2002; Ochsner et al., 2004). Importantly, two putatively different emotion regulation strategies, suppression of emotional expression and reappraisal of emotional eliciting stimuli, have been found to recruit largely overlapping frontal lobe structures in medial and bilateral dorsolateral PFC and lateral OFC (Goldin, McRae, Ramel, \& Gross, 2008).

Taken together, the research reviewed above suggests a tight link between EF and emotion regulatory ability. However, no direct evidence connecting EF and emotion regulation is available to date, so the purpose of the present research is to fill this gap by investigating whether EF is related to performance on two established laboratory tests of emotion regulation: the ability to downregulate spontaneously and the ability to downregulate when instructed to suppress emotional expressions. Next, we turn to an overview of EF measurement.

\section{Multiple EF Functions}

The need for reliable and valid measures of EF has led to the development of a number of neuropsychological tests that putatively assess dissociable facets of $\mathrm{EF}$ (Miyake, Friedman, Emerson, Witzki, \& Howerter, 2000; Royall et al., 2002). Research shows that although EF tests tend to be intercorrelated (Miyake et al., 2000), they measure different processes. In the present study, we selected tests commonly used to measure four different facets of EF-(1) working memory capacity, (2) inhibition, (3) task switching, and (4) cognitive flexibility - to determine their relationship to a laboratory-based battery of emotion regulation ability (Levenson, 2007).

Working memory refers to a set of processes that enables maintenance and manipulation of information in short-term memory in the service of a particular goal (Baddeley, 1986; Norman \& Shallice, 1986). Working memory is measured by having participants memorize and mentally manipulate groups of items (e.g., letters or numbers). Working memory might aid emotion regulation by enabling storage and manipulation of perceptual and contextual information.

Inhibition refers to the ability to suppress one behavioral response in favor of another (Cohen, Dunbar, \& McClelland, 1990). It is typically assessed by an interference procedure in which participants are instructed to respond to one aspect of a stimulus (e.g., ink color), and not to another, more perceptually salient, aspect (e.g., color word), and thereby prevent a prepotent response from occurring. Inhibition plays a role in emotion regulation by enabling suppression of the more automatic aspects of emotional responding.
Task switching refers to the ability to redirect attention quickly between tasks. It is typically measured by having participants engage in activities with alternating instruction sets (e.g., tracing mazes by alternating between letters and numbers). Task switching is important in emotion regulation because of the need to switch attention between various competing stimuli (e.g., the emotion-eliciting cue vs. the kind of emotional expression that is appropriate).

Finally, cognitive flexibility (Lezak et al., 2004; Ruff, Light, Parker, \& Levin, 1997) is the ability to generate responses quickly in keeping with predetermined criteria. Cognitive flexibility is typically measured with fluency tasks (Lezak et al., 2004) that require the participant to continually (e.g., for $1 \mathrm{~min}$ ) generate lists of words or objects that fit some criteria (e.g., words starting with the letter A). Cognitive flexibility is important for emotion regulation because of the need to remember rules governing appropriate emotional expression, devise regulatory plans, and maintain and monitor emotional responding.

\section{The Present Study: EF and Emotion Regulation}

The present study was designed to determine whether the four aspects of EF reviewed above (working memory, inhibition, task switching, and cognitive flexibility) are related to the ability to downregulate emotional responding either spontaneously or following instructions to suppress emotional responses. EF was measured using standard neuropsychological testing procedures. Emotion regulation was measured in the laboratory using procedures that generate strong emotions and create conditions designed to assess ability to downregulate emotional response (Levenson, 2007). Although prior studies have found that certain EF tests predict emotion and self-regulatory success in socially complex situations (Payne, 2005; von Hippel \& Gonsalkorale, 2005; von Hippel et al., 2000), there have been no previous examinations of the relationship between $\mathrm{EF}$ tests and the ability to downregulate emotional responses.

To ensure that participants had a wide range of EF, we included patients with neurodegenerative brain diseasesfrontotemporal lobar degeneration (FTLD) or Alzheimer's disease (AD) - as well as neurologically normal controls. Deficits in EF have been documented in dementing disorders that impact the frontal lobes (Baddeley, 1986; Duke \& Kaszniak, 2000). FTLD is characterized by neuronal loss in the frontal and anterior temporal lobes (Kertesz, Davidson, \& Munoz, 1999; Neary et al., 1998). Although degeneration in $\mathrm{AD}$ typically starts in medial temporal areas, it often spreads to include the frontal lobes (Tikofsky, Hellman, \& Parks, 1993). Thus, patients with these dementias were expected to manifest varying degrees of $\mathrm{EF}$ deficits to a greater extent than were the controls.

The emotion regulation battery used a very simple emotion elicitor, an aversive acoustic startle stimulus, so that the dementia patients would not have difficulties with interpretation and processing (Levenson, 2007). This stimulus is known to produce a strong, defensive response consisting of reflexive body movements that serve to protect the torso and head, and emotional facial behaviors, including surprise and fear (Ekman, Friesen, \& Simons, 1985; Hagemann, Levenson, \& Gross, 2006; Keltner \& Ekman, 
1996; Sturm, Rosen, Allison, Miller, \& Levenson, 2006). We and others have used similar startle stimuli in studies of emotional reactivity and regulation (e.g., Ekman et al., 1985; Hagemann et al., 2006; Roberts et al., 2004; Soto, Levenson, \& Ebling, 2005).

In the present study, we introduced the acoustic startle stimulus under three different instructional conditions designed to create different levels of demand for downregulating emotional behavior and thus test different emotion regulatory strategies. In an unwarned condition, the startle appeared without warning and participants were not given any instructions about regulating their emotional response. This condition did not impose regulatory demands and was included to measure differences in baseline reactivity to the stimuli. In a warned only condition, the startle appeared at the end of a 20 -sec countdown period and, again, participants were not given any instructions about regulating their emotional response. This condition was included to tap into spontaneous attempts to regulate emotions (Ekman et al., 1985) and was designed to mobilize the participants' own choices of regulatory strategy. In a warned with instructions to suppress condition, the startle appeared at the end of a 20 -sec countdown period and participants were explicitly instructed to hide their emotional responses.

On the basis of the role that EF plays in emotion regulation, we hypothesized that deficits in EF would be associated with poor ability to downregulate emotional responding in all groups (patients and controls). We did not expect to find this relationship in the unwarned condition, but did expect to find it in the two warned conditions (the warning period provides ample opportunity for formulating and activating regulation strategies of one's choice and for formulating plans to suppress emotional behavior). By including the two different warned conditions, we were able to examine the relationship between EF and two kinds of emotion regulation. In the warned only condition, we were able to assess spontaneous emotion regulation, in that participants were not explicitly told to downregulate. Furthermore, if they chose to downregulate they were free to do so in any way they wanted. In the warned with instructions to suppress condition, we were able to assess the ability to comply with instructions to downregulate in a particular way (using expressive suppression; Gross \& Levenson, 1993). Existing research provided a basis for predicting that EF measures would be related to the ability to downregulate spontaneously (e.g., von Hippel
\& Gonsalkorale, 2005) as well as when instructed to suppress (e.g., Goldin et al., 2008).

Given the lack of research on the connection between laboratory tests of emotion regulation and $\mathrm{EF}$, we did not make any strong predictions about which particular EF tests would be most strongly related to downregulatory ability. However, given the complexities involved with downregulating emotion in this kind of seminaturalistic situation, we speculated that tests of EF that reflected more complex processing (e.g., verbal fluency) would show the strongest relationships with downregulatory ability.

\section{METHOD}

\section{Participants}

Twenty-four FTLD and 7 AD patients were recruited through the Memory and Aging Center at the University of California, San Francisco (UCSF). Seventeen neurologically normal control participants were selected through newspaper ads. Participants were evaluated by means of clinical interviews, questionnaires, MRI structural brain scans, and neuropsychological measures (including EF tests). FTLD diagnoses were determined using the Neary clinical criteria (Neary et al., 1998). AD diagnoses were determined with the assistance of the National Institute of Neurological and Communication Diseases and by using stroke/AD and related disorders association criteria (McKhann et al., 1984). Control participants were free of neurological and psychiatric symptoms. Demographics, and the neurological status of the participants, are described in Table 1. Despite attempts to match the three groups agewise, control participants in the sample were significantly older than FTLD patients; therefore, age was used as a covariate in all analyses.

\section{EF Tests}

A trained staff at UCSF administered the EF tests. Descriptive statistics and correlations among these measures are presented in Table 2.

Digit and spatial span. To assess working memory, the digit span and its visual analogue, the spatial span, subscales (forward and backward) of the Wechsler Adult Intelligence Scale (Version III; Wechsler, 1997) were administered. Scores were summed across the correctly completed trials on the digit and spatial span trials to create a single composite score, with higher scores indicating greater working memory capacity. Reliabilities of both the digit and spatial span subscales are high, having test-retest correlations of .83 and 71, respectively (Wechsler, 1997).

Stroop. To assess inhibition, the Stroop task from the DelisKaplan Executive Function System (D-KEFS; Delis, Kaplan, \& Kramer, 2001) was used. In this task, there are two separate trials; color words are printed in either incongruent ink colors (e.g., the word "red" printed in green ink) or congruent ink colors (e.g., the word "red" printed in red ink) and the participant is asked to name

Table 1

Demographics and Dementia Status of the Sample

\begin{tabular}{|c|c|c|c|c|c|c|c|}
\hline & \multicolumn{2}{|c|}{$\begin{array}{l}\text { Normal Aging } \\
\quad(n=17 \\
\quad 8 \text { male })\end{array}$} & \multicolumn{2}{|c|}{$\begin{array}{c}\mathrm{AD} \\
(n=7 \\
5 \text { male })\end{array}$} & \multicolumn{2}{|c|}{$\begin{array}{l}\text { FTLD } \\
(n=24, \\
18 \text { male })\end{array}$} & \multirow[b]{2}{*}{ Test Statistics } \\
\hline & $M$ & $\frac{S D}{S D}$ & $M$ & $S D$ & $M$ & $\overline{S D}$ & \\
\hline $\begin{array}{l}\text { Age } \\
\text { MMSE }\end{array}$ & $\begin{array}{l}66.84_{b} \\
29.7_{\mathrm{a}}\end{array}$ & $\begin{array}{l}8.52 \\
0.47 \\
\end{array}$ & $\begin{array}{l}63.56_{\mathrm{b}} \\
22.86_{\mathrm{b}}\end{array}$ & $\begin{array}{l}7.10 \\
4.02 \\
\end{array}$ & $\begin{array}{l}60.22_{\mathrm{a}} \\
26.16_{\mathrm{a}} \\
\end{array}$ & $\begin{array}{l}7.13 \\
3.08 \\
\end{array}$ & $\begin{array}{l}\chi^{2}(2, N=48)=3.56, \text { n.s. } \\
F(2,45)=3.74, p<.05 \\
F(2,45)=13.54, p<.05\end{array}$ \\
\hline
\end{tabular}

Note-Groups with different subscripts differed from each other at $p<.05$. AD, Alzheimer's disease; FTLD, frontotemporal lobar degeneration; MMSE, Mini-Mental State Examination scores (Folstein, Folstein, \& McHugh, 1975), which provide information about the patients' overall impairment from dementia and indicate that $\mathrm{AD}$ patients exhibited the most impairment. 
Table 2

Descriptive Statistics and Intercorrelations Among EF Measures

\begin{tabular}{lcccccr}
\hline & $M$ & $S D$ & 1 & 2 & 3 & 4 \\
\hline 1. Working memory & 21.79 & 3.90 & - & & & \\
2. Stroop (inhibition; residualized) & 0 & 27.22 & $-.47^{*}$ & - & & \\
3. Trail making (task switching; residualized) & 0 & 45.32 & -.05 & .19 & - & \\
4. Verbal fluency (cognitive flexibility) & 33.62 & 15.38 & $.27^{*}$ & -.15 & -.10 & - \\
\hline
\end{tabular}

Note $-N=48$. Uncorrected mean reading time on the critical Stroop inference card was $79.66 \mathrm{sec}$ $(S D=37.55)$. Uncorrected mean time to completion on the numbers-to-letters trail-making card was $131.15 \sec (S D=66.87) . \quad{ }^{*} p<.05$ or below.

the ink color. To control for individual differences in color naming speed, an overall inhibition score was created by predicting incongruent color word reading time from congruent color word reading time and saving the residuals. ${ }^{1}$ These residual scores were then used as a measure of inhibition with longer times, higher scores indicating poorer inhibition. Reliability of the Stroop is reasonable, with test-retest correlations of .70-.79 (Delis et al., 2001).

Trail making. To assess task switching, the D-KEFS trail-making test was used (Delis et al., 2001). In this task, participants were instructed to alternate between connecting letters and connecting numbers printed in scrambled order on a card (connect from " $\mathrm{A}$ " to " 1 " to "B" to " 2 "). If the participant made an error, the examiner pointed it out and instructed the participant to return to the correct location while the clock kept running. To hold individual differences in number and letter processing speed constant, participants also completed two trials where the task was to connect letters to letters and numbers to numbers. To control for individual differences on the latter trials, an overall task-switching score was created by predicting time to completion on the numbers-to-numbers trials from the letters-to-letters and numbersto-numbers trials, and saving the resulting residuals. These residual scores were used as a measure of task switching, where shorter times and lower scores indicate better task switching. Reliability of the trailmaking test is reasonable, with test-retest correlations of .70-.79 (Delis et al., 2001).

Verbal fluency. To assess cognitive flexibility, the verbal fluency test (Delis et al., 2001) was used. On separate 1-min trials, participants were asked to generate words that began with the letters F, A, and S (excluding proper nouns and not repeating the same word with different suffixes). Verbal fluency was calculated as the total number of correct words produced across the three trials. More words produced indicate better cognitive flexibility. Reliability of the verbal fluency test is high, with a test-retest correlation of .90 (Delis et al., 2001).

Means on the EF variables ${ }^{2}$ are presented in Table 3 for the FTLD, $\mathrm{AD}$, and normal controls. As expected, these show lower levels of EF for patients compared with controls. This was particularly the case for verbal fluency. Additionally, Stroop scores were lower among FTLD patients than among AD patients and controls.

\section{Emotional Responding}

Two aspects of the motor response to the startle were measured: somatic activity and facial expressive behavior.

Somatic activity. General body movement associated with the startle reflex was continuously monitored with an electromagnetic transducer attached to a platform under the participant's chair. The transducer generates an electrical signal proportional to the amount of movement in any direction and is very sensitive to the kinds of head and trunk movements that occur in response to the startle stimulus. The signal from the transducer was amplified using a Grass Model 7 polygraph, processed continuously (at a $300-\mathrm{Hz}$ sampling rate), and was averaged for the $5 \mathrm{sec}$ following the startle stimulus, using software written by one of the authors (R.W.L.). A number of autonomic nervous system responses were also monitored as part of the experimental procedure, but they are not included here because our primary interest was in measuring regulation of visible motoric components of the startle response.

Facial expressive behavior. A front view of the participant's face was videotaped using a partially concealed, remotely controlled camera. A team of research assistants coded facial expressive behavior from the videotapes using the emotional expressive behavior coding system (Gross \& Levenson, 1993). Two coders who were blind to diagnostic status and experimental instructions viewed each tape without sound. Conditions were coded in randomized order. Coders rated the intensity of emotional expressions of anger, contempt, confusion, disgust, fear, happiness, embarrassment, interest, sadness, and surprise on a second-by-second basis during the $5 \mathrm{sec}$ following the startle stimulus using a 4-point intensity scale $(0=$ absent; $3=$ strong $)$. Intercoder reliability for the full set of codes was high $(\alpha=.79)$. To capture the wide variety of emotional expressions typically produced by the startle stimulus (Ekman et al., 1985; Keltner \& Ekman, 1996), a measure of overall facial expressive behavior was computed by averaging across all categories of emotional expressions.

\section{Behavioral Emotion Regulatory Strategies}

To measure emotion regulatory strategies, participants were asked an open-ended question at the end of the regulation trials; specifically, in the warned only trial, they were asked "Did you do anything different, knowing this time that the noise was coming?" After the warned with instructions to suppress trial, participants were asked to describe how they tried to hide their reactions. Their responses were transcribed and categorized by one of the authors (A.G.) into five different categories: (1) no regulation; (2) suppression of physiological and behavioral response (e.g., "held breath, held facial muscles steady"); (3) modified sensory features of the noise either internally (e.g., "paid attention to the quality of the noise") or externally (e.g., "covered head to buffer noise"); (4) cognitive reinterpretation (e.g.,

Table 3

Performance and Differences Between Groups on EF Tests

\begin{tabular}{|c|c|c|c|c|c|c|c|}
\hline & \multicolumn{2}{|c|}{$\begin{array}{l}\text { Normal Aging } \\
\quad(n=17)\end{array}$} & \multicolumn{2}{|c|}{$\begin{array}{c}\mathrm{AD} \\
(n=7)\end{array}$} & \multicolumn{2}{|c|}{$\begin{array}{l}\text { FTLD } \\
(n=24)\end{array}$} & \multirow[b]{2}{*}{ Test Statistics } \\
\hline & $M$ & $S D$ & M & $S D$ & $M$ & $S D$ & \\
\hline Working memory & 22.59 & 3.16 & 19.86 & 3.98 & 21.79 & 5.08 & n.s. \\
\hline Stroop (inhibition; residualized) & $-7.09_{\mathrm{a}}$ & 8.21 & $22.53_{\mathrm{b}}$ & 53.00 & $-1.55_{\mathrm{a}}$ & 23.25 & $F(2,45)=3.31, p<.05$ \\
\hline Trail making (task switching; residualized) & -8.01 & 42.76 & 3.83 & 50.13 & 4.56 & 46.85 & n.s. \\
\hline Verbal fluency (cognitive flexibility) & $48.71_{\mathrm{a}}$ & 8.30 & $25.71_{\mathrm{b}}$ & 11.76 & $25.25_{\mathrm{b}}$ & 11.90 & $F(2,45)=26.27, p<.05$. \\
\hline
\end{tabular}

Note-Groups with different subscripts differed from each other at $p<.05$ or below. AD, Alzheimer's disease; FTLD, frontotemporal lobar degeneration. 
"thought of the noise as a starter's pistol starting a race"); or (5) no specific regulatory strategy (e.g., "just prepared").

\section{Procedure}

Participants came to our laboratory at the University of California, Berkeley, for a single experimental session scheduled within 3 months $(M=15.44$ days, $S D=7.5)$ of their EF being assessed at $\mathrm{UCSF}^{3}$ On arrival, each participant signed the consent forms, was seated in a chair located $1.25 \mathrm{~m}$ from a 48 -cm color television monitor, and had sensors attached for physiological monitoring. The full experimental protocol consisted of a series of tasks designed to assess emotional functioning (e.g., emotion-eliciting films, recall of emotional memories; Levenson, 2007). The present article focuses on the three startle trials where participants were presented with a loud noise (115-dB 100-msec-long burst of white noise) that came through two loudspeakers located behind each participant's head. ${ }^{4}$ The sound can be likened to a "gunshot"; as noted earlier, it produces a large reflexive defensive startle reaction (Hagemann et al., 2006).

Each of the three startle trials began with a 1-min baseline period, during which the participant was instructed to view an " $\mathrm{X}$ " on the TV screen. On the first trial (unwarned), the startle occurred without warning at the end of the 1-min baseline period. On the second trial (warned only), participants were told by the experimenter and on the television screen that the startle stimulus would occur at the end of a 20 -sec countdown presented on the television screen. Under these conditions, most people spontaneously downregulate their emotional response, bracing for the upcoming aversive stimulus (Ekman et al., 1985). On the third trial (warned with instructions to suppress), participants were told that the startle stimulus would occur at the end of the countdown. They were also told "This time, if you have any reaction as you hear the noise, try your best not to let your feelings show. Pretend someone is watching you, and you do not want them to see that you are reacting to the noise."

We used these three instructional conditions with the acoustic startle in a number of previous studies with patients and normals (Hagemann et al., 2006; Roberts et al., 2004; Soto et al., 2005; Sturm et al., 2006), and generally used them in the same fixed order (unwarned, warned only, warned with instructions to suppress). To get a relatively pure measure of reactivity to the startle when it occurred without prior warning, the unwarned condition had to be administered first. For the two warned conditions, our desire to get an estimate of spontaneous regulation ability required the warned only condition to come next (because the warned with instructions to suppress condition would cue the participant to use a suppression strategy on subsequent trials).

\section{Reliability of the Emotion Regulation Battery}

To assess the reliability of the emotion regulation battery, we examined data from a small sample of 9 dementia patients and normal controls who returned to our laboratory and repeated the battery approximately 1 year after their initial visit. Reliabilities for the overall emotional responses (aggregated body movement and emotional facial response averaged over the three startle conditions) were quite high (the test-retest correlation was .74). Examining the three startle conditions separately, reliabilities were high for the unwarned and warned only conditions (test-retest correlations of .71 and .70, respectively), and moderate for the warned with instructions to suppress condition (the test-retest correlation was .55). Within the limits of our small reliability sample, we believe that these data suggest that, even over this relatively long time interval, the emotion regulation battery has quite reasonable levels of reliability.

\section{Data Reduction and Preliminary Analyses}

For each startle trial, the somatic activity and facial expressive averages for the $5 \mathrm{sec}$ following the startle burst were normalized and an emotional responding composite score was calculated by averaging the two normalized values. This emotional responding composite was used as the criterion variable in all analyses. Statistical analyses were performed using the SAS statistical package (Ver- sion 9; SAS Institute Inc., Cary, NC). To examine the relationship between EF and emotional responding, data from all participants were pooled (this maximized variability in EF). Where significant relationships were found between EF and emotional responding, we followed up with an analysis that tested the interaction of diagnostic group and EF scores to determine whether diagnostic group moderated the relationship between EF and emotional responding.

To determine whether the experimental manipulation used to produce emotional responding was effective, we tested reduction in somatic activity and facial expressive behavior separately for the two warned trials, compared with the unwarned trial, using pairedsample $t$ tests. Results for somatic activity showed reduction from the unwarned to the warned only condition [unwarned, $M=3.37$, $S D=2.33$; warned only, $M=2.07, S D=1.97 ; t(47)=5.16, p<$ $.05]$ and from the unwarned to the warned with instructions to suppress trial [warned with instructions to suppress, $M=1.49, S D=$ $1.24 ; t(47)=5.76, p<.05]$. Results for facial expressive behavior showed reduction from the unwarned to the warned only trial [unwarned, $M=0.67, S D=0.57$; warned only, $M=0.43, S D=0.54$; $t(47)=2.80, p<.05]$ and from the unwarned to the warned with instructions to suppress trial [warned with instructions to suppress, $M=0.27, S D=0.50 ; t(47)=3.85, p<.05]$. These results suggest that the unwarned condition elicited more emotional behaviors both on the face and in somatic activity than did either the warned only or warned with instructions to suppress trials.

\section{Behavioral Emotion Regulatory Strategies}

Open-ended responses were analyzed for both regulation trials to elucidate the type of emotion regulatory strategies used in each condition. In the warned only trial, $49 \%$ of participants reported not using a strategy. Among those who reported using a strategy, $12.5 \%$ reported using suppression, $16.67 \%$ reported using attention distraction, $4.17 \%$ reported using cognitive reinterpretation, and $66.67 \%$ did not provide detailed enough information to reliably categorize their emotion regulatory strategy. Thus, reflecting the lack of specific instructions, this condition produced a wide range of reported strategies (and lack thereof). In contrast, in the warned with instructions to suppress trial, $97 \%$ reported using a regulatory strategy. Of these, $56.82 \%$ reported using suppression, $20.45 \%$ reported using attention distraction, and $22.73 \%$ of the participants did not provide enough detail to categorize the emotional regulatory strategy reliably. Thus, in this condition most participants appeared to follow the instructions and used the prescribed suppression strategy.

\section{RESULTS}

\section{EF and Unwarned Startle Trial}

We hypothesized that EF would be related to emotional responding on the two warned trials, which created a demand for and provided time for downregulation, but not to responses on the unwarned trial. Consistent with this prediction, we found no significant correlations between the four EF functions and emotional responding on the unwarned trial; partial correlations (controlling for age) were: working memory, $r(46)=.09$, n.s.; Stroop, $r(46)=$ .07 , n.s.; trail making, $r(46)=.00$, n.s.; and verbal fluency, $r(46)=-.01$, n.s.

\section{EF and Emotion Regulation Trials}

Analysis. We hypothesized that deficits in EF would be associated with deficits in emotion regulation on the warned trials. Because the warned only and warned with instructions to suppress trials differed in how explicit the demand for regulation was, we considered each separately. ${ }^{5}$ Specifically, for each trial, we conducted a series 
Table 4

Standard Regression Coefficients and $\boldsymbol{t}$ Statistics of EF Predicting Emotional Behavior in the Regulation Trials

\begin{tabular}{|c|c|c|c|c|}
\hline & \multicolumn{2}{|c|}{ Warned Only } & \multicolumn{2}{|c|}{$\begin{array}{l}\text { Warned With } \\
\text { Instructions } \\
\text { to Suppress }\end{array}$} \\
\hline & $\beta$ & $t$ & $\beta$ & $t$ \\
\hline Working memory & -.15 & -1.23 & .09 & 0.59 \\
\hline Stroop (inhibition; residualized) & .04 & 0.74 & .01 & 0.97 \\
\hline Trail making (task switching; residualized) & -.07 & -0.57 & .16 & 0.27 \\
\hline Verbal fluency (cognitive flexibility) & $-.31^{*}$ & $-2.44^{*}$ & $-.39^{*}$ & $-2.75^{*}$ \\
\hline
\end{tabular}

Note-Emotional responding in the unwarned trial and age were held constant in all analyses, $F(1,44) . \quad$ *Bonferroni-adjusted $p<.025$ or below.

of four separate regression analyses, each with one EF test as the predictor variable, our composite measure of emotional responding as the criterion, and age and emotional responding on the unwarned trial as covariates. Controlling for emotional responding on the unwarned trial enabled us to examine emotional behavior in the two regulation trials free of any individual differences in the magnitude of emotional responding (Ekman et al., 1985). To control for multiple statistical tests, we applied a Bonferroni adjustment and adopted a more conservative significance level of .025 for a priori one-tailed tests.

Results. For the warned only trial, the only EF measure that predicted emotional responding was verbal fluency, such that higher verbal fluency scores were related to more reduction in emotional responding $[F(1,45)=$ 7.77, $p<.05, \beta=-.31]$. Results of these analyses for all four EF measures are presented in Table 4. No significant interactions were found between verbal fluency and diagnostic group membership $(F<1) .{ }^{6}$ Furthermore, we also examined the unique predictive ability of verbal fluency above and beyond the other three measures of EF, using a simultaneous regression analysis. This revealed that the relationship between verbal fluency and emotional responding in the warned only trial was somewhat

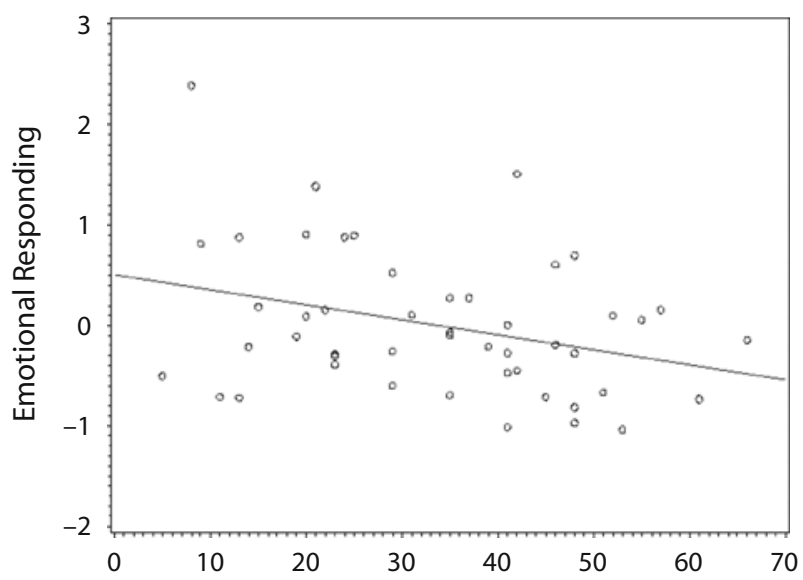

Figure 1. Residualized values of emotional responding in the warned only trial as a function of verbal fluency performance after controlling for age and emotional responding in the unwarned startle trial. Note: Lower values indicate more reduction in emotional responding. attenuated, but remained significant $[F(1,41)=4.92, p<$ $.05, \beta=-.28]$ after the effects of the other three EF tests were held constant.

For the warned with instructions to suppress trial, again, the only EF measure that predicted emotional responding was verbal fluency, such that higher verbal fluency scores were related to more reduction in emotional responding $[F(1,45)=7.97, p<.05, \beta=-.39]$. Results of these analyses for all four EF measures are presented in Table 4. No significant interactions were found between verbal fluency and diagnostic group membership $(F<1) .^{7}$ Finally, we also examined the unique predictive ability of verbal fluency above and beyond the other three measures of EF, using a simultaneous regression analysis. This analysis revealed that the relationship between verbal fluency and emotional responding on the warned with instructions to suppress trial remained significant when controlling for the other three measures of $\operatorname{EF}[F(1,41)=8.48, p<.05$, $\beta=-.44]$.

Because outliers can unduly influence findings with small sample sizes, we examined regression scatterplots (verbal fluency vs. residualized emotional responding) after controlling for age and for emotional responding on the unwarned trial for both the warned only (Figure 1)

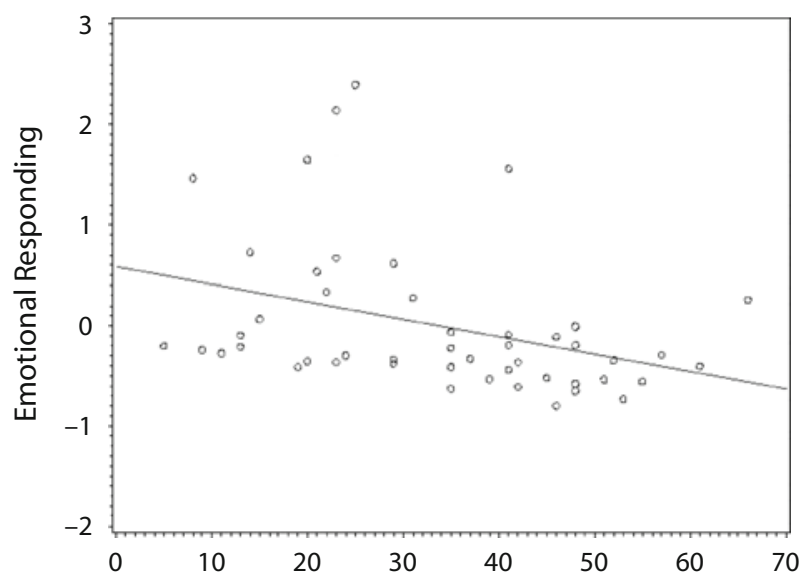

Figure 2. Residualized values of emotional responding in the warned with instructions to suppress trial as a function of verbal fluency performance after controlling for age and emotional responding in the unwarned startle trial. Note: Lower values indicate more reduction in emotional responding. 
and warned with instructions to suppress (Figure 2) trials. Both visual examination and formal outlier analyses (i.e., rerunning the analyses after excluding any observation that had a Cook's $D$ greater than $4 / N$ ) indicated that outliers were not responsible for the findings.

\section{DISCUSSION}

This study tested the hypothesis that deficits in EF, which are believed to underlie goal-directed behavior and regulation, would be associated with deficits in emotion regulation. Using a sample of patients (FTLD and AD) and neurologically healthy controls with a wide range of EF and an aversive acoustic startle stimulus known to produce sizeable emotional responding, we found support for this hypothesis both when the demand for regulation was implicit and participants used spontaneously recruited emotion regulatory strategies (our warned only condition), and when it was explicit and participants were instructed to suppress their responses (our warned with instructions to suppress condition). Importantly, the following two factors endowed this finding with considerable specificity: (1) EF only predicted emotional responding on the regulation trials, and not when the startle stimulus occurred unexpectedly without a warning; and (2) of four commonly used measures of EF-working memory, Stroop, trail making, and verbal fluency-only deficits in verbal fluency (a measure of cognitive flexibility) predicted deficits in emotion regulation.

We had predicted that EF deficits would not be related to emotional responding to the unwarned startle. This prediction was based on the view that emotional reactivity to simple stimuli such as the acoustic startle are largely subserved by brain stem and limbic circuits (Davis, Gendelman, Tischler, \& Gendelman, 1982), which are not assessed by EF measures. Additionally, we had predicted that $\mathrm{EF}$ deficits would be related to emotional regulation in the two regulation conditions, both when participants were free to use a regulatory strategy of their choice spontaneously, and in the suppression condition, when participants are given explicit instructions to use suppression as an emotion regulatory strategy. This prediction was based on the view that emotion regulation is subserved by frontal-subcortical circuits (Roberts et al., 2004; Rolls, 2000; Stuss \& Levine, 2002), which are assessed by EF measures. Together, these findings are evidence of a differentiated view of emotional circuitry in the brain - namely, that there are different circuits for emotional responses and for regulating these responses.

It may seem surprising that among EF measures only verbal fluency predicted emotion regulation, but this underscores the fact that putative measures of EF are not interchangeable; rather, they capture clinically, functionally, and anatomically different aspects of EF (Miyake et al., 2000; Royall et al., 2002). For example, the Stroop test, which assesses inhibition of a dominant response, might have been viewed as the most likely candidate for predicting emotion regulation. Stroop performance failing to correlate with emotion regulation underscores the fact that the ability to mount a successful emotion regulatory response to an aversive auditory stimulus, both spontaneously and to suppress when instructed, involves more than just response inhibition. In our view, successful emotion regulation draws on a complex set of skills, including strategy formulation, behavioral monitoring, evaluation, and adjustment. Viewed from this perspective, there are similarities between the process of EF, as indexed by generating words of a certain kind on the verbal fluency task, and downregulating an emotion, insofar as both tests require the individual to be able to devise an advantageous strategy, to monitor ongoing performance, and to inhibit erroneous responses (Lezak et al., 2004; Shimamura, 2000; Troyer, Moscovitch, \& Winocur, 1997).

Although emotion downregulation to an acute aversive stimulus is arguably highly prototypical, there clearly are other kinds of emotion regulation and kinds of stimuli that occasion regulation. Additional research will be necessary to determine whether EF as measured by tests of verbal fluency is associated with the ability to engage in other kinds of emotion regulation (e.g., amplification, cognitive reappraisal, and attentional control) and other kinds of stimuli (e.g., those that are more chronic, those that evoke different kinds of emotion).

In summary, we were able to present evidence that the relationship between verbal fluency and EF was consistent across the three groups and across two different emotion regulatory conditions. Thus, we have confidence that these findings establish a true link between measures of verbal fluency and these two forms of emotion regulation. Furthermore, we demonstrated that the findings do not merely result from the measures associated with diagnosis, and we found that findings hold robust across two different emotion regulatory conditions.

\section{Strengths and Limitations}

This study bridges the domains of cognitive and emotional functioning using a novel methodology that links neuropsychological tests of $\mathrm{EF}$ with emotional regulation in both neurological patients and normal controls. Because EF was measured using performance-based neuropsychological tests, and emotion regulation was assessed using laboratory measures of emotional facial behavior and somatic muscle activity, concerns about shared method variance were minimized.

The study has several limitations that may have affected our findings. We tested only a subset of the available tests of EF and only a single emotion elicitor. Thus, our findings might have differed with other tests (e.g., $N$-back, Wisconsin Card Sort) and other elicitors (e.g., films, pictures). To provide a wide range of EF, we included patients with two kinds of dementia, as well as neurologically normal participants, so our findings may not generalize to other neurological populations. Additionally, because the three startle conditions occurred in a fixed order, we cannot rule out order or habituation effects. Furthermore, individual patterns of neurodegeneration have not been characterized for the patients in this sample; therefore, we cannot directly determine relationships between regional neural loss and deficits in our EF and emotion regulation measures. The fact that no diagnostic group differences 
emerged in our analyses could be due to small sample size, especially in the AD group. Finally, as noted above, our results establish a link between verbal fluency and the ability to downregulate the emotional response to an acute aversive stimulus spontaneously and when instructed to suppress expressive responses. Thus, we cannot know the extent to which these findings generalize to other kinds of emotion regulation and to other emotional stimuli.

\section{CONCLUSION}

This study provided the first evidence that an aspect of EF (cognitive flexibility as measured by a test of verbal fluency) predicts the ability to downregulate emotional responses, both when the demand was implicit and when it was explicit to suppress. These findings provide a behavioral counterpoint to imaging studies that show an important inhibitory relationship between frontal circuits and key emotion centers in the brain (e.g., medial prefrontal and amygdala circuits; Ochsner et al., 2002). EF predicted both spontaneous emotion regulation and instructed suppression when participants knew when the stimulus would occur, but did not predict emotional responding when they did not; and only a test of verbal fluency, and not tests of other aspects of EF including working memory, inhibition, and task switching, predicted that emotion regulation underscores the precise nature of these relationships and the importance of using differentiated measures of EF and emotional functioning.

\section{AUTHOR NOTE}

This research was supported by National Institute on Aging Grants AG17766 and P01-AG19724 (subcontract) and National Institute of Mental Health Grant T32 MH20006 awarded to R.W.L.; National Institute on Aging Grants 1K08AG02076001, AG10129, P50-AG05142, and AG16570 awarded to B.L.M.; and the Alzheimer's Disease Research Center of California. We thank the members of the Berkeley Psychophysiology Laboratory and the Memory and Aging Center for their help in conducting this research. Address correspondence to A. Gyurak or R. W. Levenson, Institute of Personality and Social Research, Department of Psychology, University of California, 4143 Tolman Hall MC 5050, Berkeley, CA 94720-5050 (e-mail: agyurak@berkeley.edu, boblev@socrates.berkeley.edu).

\section{REFERENCES}

BAdDEley, A. (1986). Working memory. New York: Oxford University Press.

Banfield, J. F., Wyland, C. L., Macrae, C. N., Münte, T. F., \& HeathERTON, T. F. (2004). The cognitive neuroscience of self-regulation. In R. F. Baumeister \& K. D. Vohs (Eds.), Handbook of self-regulation: Research, theory, and applications (pp. 62-83). New York: Guilford.

Beauregard, M., Lévesque, J., \& Bourgouin, P. (2001). Neural correlates of conscious self-regulation of emotion. Journal of Neuroscience, 21, RC165:1-6.

Cohen, J. D., Dunbar, K., \& McClelland, J. L. (1990). On the control of automatic processes: A parallel distributed processing account of the Stroop effect. Psychological Review, 97, 332-361.

Davis, M., Gendelman, D. S., Tischler, M. D., \& Gendelman, P. M. (1982). A primary acoustic startle circuit: Lesion and stimulation studies. Journal of Neuroscience, 2, 791-805.

Delis, D. C., Kaplan, E., \& Kramer, J. H. (2001). The Delis-Kaplan Executive Function System. San Antonio: Psychological Corporation.

Denckla, M. B. (1996). A theory and model of executive function: A neuropsychological perspective. In G. R. Lyon \& N. A. Krasnegor
(Eds.), Attention, memory, and executive function (pp. 263-278). Baltimore: Paul H. Brookes.

Duke, L. M., \& KaszniaK, A. W. (2000). Executive control functions in degenerative dementias: A comparative review. Neuropsychology Review, 10, 75-99.

Eigsti, I.-M., Zayas, V., Mischel, W., Shoda, Y., Ayduk, O., Dadlani, M. B., ET AL. (2006). Predicting cognitive control from preschool to late adolescence and young adulthood. Psychological Science, 17, 478-484.

Ekman, P., Friesen, W. V., \& Simons, R. C. (1985). Is the startle reaction an emotion? Journal of Personality \& Social Psychology, 49, 1416-1426.

Eslinger, P. J., \& DAmasio, A. R. (1985). Severe disturbance of higher cognition after bilateral frontal lobe ablation: Patient EVR. Neurology, 35, 1731-1741.

Folstein, M. F., Folstein, S. E., \& McHugh, P. R. (1975). Mini-mental state: A practical method for grading the cognitive state of patients for the clinician. Journal of Psychiatric Research, 12, 189-198.

Goldin, P. R., McRae, K., RAmel, W., \& Gross, J. J. (2008). The neural bases of emotion regulation: Reappraisal and suppression of negative emotion. Biological Psychiatry, 63, 577-586.

Gross, J. J., \& LeVenson, R. W. (1993). Emotional suppression: Physiology, self-report, and expressive behavior. Journal of Personality \& Social Psychology, 64, 970-986.

Gross, J. J., \& Thompson, R. A. (2007). Emotion regulation: Conceptual foundations. In J. J. Gross (Ed.), Handbook of emotion regulation (pp. 3-24). New York: Guilford.

Hagemann, T., Levenson, R. W., \& Gross, J. J. (2006). Expressive suppression during an acoustic startle. Psychophysiology, 43, 104-112.

Jensen, A. R., \& Rohwer, W. D., JR. (1966). The Stroop color-word test: A review. Acta Psychologica, 25, 36-93.

Keltner, D., \& Ekman, P. (1996). Affective intensity and emotional responses. Cognition \& Emotion, 10, 323-328.

Kertesz, A., Davidson, W., \& Munoz, D. G. (1999). Clinical and pathological overlap between frontotemporal dementia, primary progressive aphasia and corticobasal degeneration: The Pick complex. Dementia \& Geriatric Cognitive Disorders, 10(Suppl. 1), 46-49.

LEVENSON, R. W. (2007). Emotion elicitation with neurological patients. In J. A. Coan \& J. J. B. Allen (Eds.), Handbook of emotion elicitation and assessment (pp. 158-168). New York: Oxford University Press.

LezaK, M. D., Howieson, D. B., Loring, D. W., Hannay, H. J., \& FisCHER, J. S. (2004). Neuropsychological assessment (4th ed.). New York: Oxford University Press.

McKhann, G., Drachman, D., Folstein, M., Katzman, R., Price, D., \& Stadlan, E. M. (1984). Clinical diagnosis of Alzheimer's disease: Report of the NINCDS-ADRDA work group under the auspices of the Department of Health and Human Services task force on Alzheimer's disease. Neurology, 34, 939-944.

Miyake, A., Friedman, N. P., Emerson, M. J., Witzki, A. H., \& HowERTER, A. (2000). The unity and diversity of executive functions and their contributions to complex "frontal lobe" tasks: A latent variable analysis. Cognitive Psychology, 41, 49-100.

Neary, D., Snowden, J. S., Gustafson, L., Passant, U., Stuss, D., BLACK, S., ET AL. (1998). Frontotemporal lobar degeneration: A consensus on clinical diagnostic criteria. Neurology, 51, 1546-1554.

Norman, D. A., \& Shallice, T. (1986). Attention to action: Willed and automatic control of behavior. In R. J. Davidson, G. E. Schwartz, \& D. Shapiro (Eds.), Consciousness and self-regulation: Advances in research and theory (Vol. 4, pp. 1-18). New York: Plenum.

OChSNer, K. N., Bunge, S. A., Gross, J. J., \& Gabrieli, J. D. E. (2002). Rethinking feelings: An fMRI study of the cognitive regulation of emotion. Journal of Cognitive Neuroscience, 14, 1215-1229.

OChSNER, K. N., RAY, R. D., COOPER, J. C., RobertSON, E. R., Chopra, S., Gabrieli, J. D. E., \& Gross, J. J. (2004). For better or for worse: Neural systems supporting the cognitive down- and up-regulation of negative emotion. NeuroImage, 23, 483-499.

PAYNe, B. K. (2005). Conceptualizing control in social cognition: How executive functioning modulates the expression of automatic stereotyping. Journal of Personality \& Social Psychology, 89, 488-503.

Posner, M. I., \& Rothbart, M. K. (2000). Developing mechanisms of self-regulation. Development \& Psychopathology, 12, 427-441.

Roberts, N. A., Beer, J. S., Werner, K. H., ScAbini, D., Levens, S. M., 
Knight, R. T., \& LeVenson, R. W. (2004). The impact of orbital prefrontal cortex damage on emotional activation to unanticipated and anticipated acoustic startle stimuli. Cognitive, Affective, \& Behavioral Neuroscience, 4, 307-316.

Rolls, E. T. (2000). Memory systems in the brain. Annual Review of Psychology, 51, 599-630.

Royall, D. R., Lauterbach, E. C., Cummings, J. L., Reeve, A., RumMANS, T. A., KAUfER, D. I., ET AL. (2002). Executive control function: A review of its promise and challenges for clinical research. A report from the committee on research of the American Neuropsychiatric Association. Journal of Neuropsychiatry \& Clinical Neurosciences, 14, 377-405.

Ruff, R. M., Light, R. H., Parker, S. B., \& Levin, H. S. (1997). The psychological construct of word fluency. Brain \& Language, 57, 394-405.

Rule, R. R., Shimamura, A. P., \& Knight, R. T. (2002). Orbitofrontal cortex and dynamic filtering of emotional stimuli. Cognitive, Affective, \& Behavioral Neuroscience, 2, 264-270.

Shimamura, A. P. (2000). Toward a cognitive neuroscience of metacognition. Consciousness \& Cognition, 9, 313-323.

Smith, E. E., \& Jonides, J. (1999). Storage and executive processes in the frontal lobes. Science, 283, 1657-1661.

Soto, J. A., Levenson, R. W., \& Ebling, R. (2005). Cultures of moderation and expression: Emotional experience, behavior, and physiology in Chinese Americans and Mexican Americans. Emotion, 5, 154-165.

Sturm, V. E., Rosen, H. J., Allison, S., Miller, B. L., \& Levenson, R. W. (2006). Self-conscious emotion deficits in frontotemporal lobar degeneration. Brain, 129, 2508-2516.

Stuss, D. T., \& Alexander, M. P. (2000). Executive functions and the frontal lobes: A conceptual view. Psychological Research, 63, 289-298.

Stuss, D. T., \& Levine, B. (2002). Adult clinical neuropsychology: Lessons from studies of the frontal lobes. Annual Review of Psychology, 53, 401-433.

Tikofsky, R. S., Hellman, R. S., \& Parks, R. W. (1993). Single photon emission computed tomography and applications to dementia. In R. W. Parks, R. F. Zec, \& R. S. Wilson (Eds.), Neuropsychology of Alzheimer's disease and other dementias (pp. 489-510). New York: Oxford University Press.

Troyer, A. K., Moscovitch, M., \& Winocur, G. (1997). Clustering and switching as two components of verbal fluency: Evidence from younger and older healthy adults. Neuropsychology, 11, 138-146.

von HiPPel, W., \& Gonsalkorale, K. (2005). "That is bloody revolting!" Inhibitory control of thoughts better left unsaid. Psychological Science, 16, 497-500. von Hippel, W., Silver, L. A., \& Lynch, M. E. (2000). Stereotyping against your will: The role of inhibitory ability in stereotyping and prejudice among the elderly. Personality \& Social Psychology Bulletin, 26, 523-532.

WechsLer, D. (1997). Wechsler Adult Intelligence Scale (3rd ed.). San Antonio: Harcourt Assessment.

Zelazo, P. D., \& Cunningham, W. A. (2007). Executive function: Mechanisms underlying emotion regulation. In J. J. Gross (Ed.), Handbook of emotion regulation (pp. 135-158). New York: Guilford.

\section{NOTES}

1 . There are a variety of ways used to calculate interference (Jensen $\&$ Rohwer, 1966). We chose residualized scores over other methods because this resulted in a distribution that best approximated normality.

2. Examination of the distribution ( $S D$ and variance) and skewness, a measure of asymmetry, suggest that all four measures of EF used in the present analyses approximate normality and have sufficient spread.

3. Repeating our major analyses using the days between the two assessments as a covariate did not change any of the reported findings.

4. The paradigm employed here is different from the emotion modulated startle paradigm, which uses repeated presentations of a lower amplitude noise (typically $95-105 \mathrm{~dB}$ ).

5. We considered the possibility that forewarning participants about the impending startle noise might have interacted with $\mathrm{EF}$ and diagnosis. Thus, we created facial behavior and body movement composite scores for the 20-sec countdown leading up to the startle in the warned only and warned with instructions to suppress trials. We then conducted a set of analyses similar to those reported in the main analyses with these new composite scores as the dependent measures. These analyses confirmed the general findings reported here-namely, that higher verbal fluency was related to lower levels of facial behavior and body movement during the anticipatory period. No relationship was found for the other three measures of EF and there were no interactions with patient status or diagnosis.

6. Controlling for diagnostic group membership attenuated the relationship between verbal fluency and emotional responding on the warned only trial $[F(5,42)=3.35, p=.07]$.

7. Controlling for diagnostic group membership reduced the relationship between verbal fluency and emotional responding on the warned with instructions to suppress trial to nonsignificant levels $[F(1,42)=$ 2.73 , n.s.].

(Manuscript received January 29, 2008; revision accepted for publication November 7, 2008.) 